\title{
A Method for Ranking Fuzzy Numbers and Its Application to Game with Fuzzy Profit
}

\author{
Yingchao Shao Zheng Pei \\ School of Mathematics \& Computer Science, Xihua University, Chengdu 610039, China
}

\begin{abstract}
In this paper, a new method for ranking fuzzy numbers is proposed. Based on decomposition theorem of fuzzy set, firstly, the method considers interval numbers, the relation between lines and interval numbers is discussed in Cartesian coordinates, the distance of $\operatorname{dot} A$ to lines $l$ is used as evaluation of interval number. Then, integral of all distances is used as evaluation of fuzzy number. Finally, ranking fuzzy numbers is implemented by the evaluations of fuzzy numbers. In order to illustrate the ranking method, numeric example is shown, and for the comparative study, our method is compared with four existing ranking methods. As an example of potential applications, the proposed method is applied to game with Fuzzy Profit.
\end{abstract}

Keywords: Interval numbers, Fuzzy numbers, Rank, Game with Fuzzy Profit

\section{Introduction}

Fuzzy numbers are an important issue in research on the fuzzy set theory. Because of the suitability for representing uncertain values, fuzzy numbers have been widely used in many applications. Since fuzzy numbers represent uncertain numeric values, it is difficult to rank them according to their magnitude, Many methods for ranking fuzzy numbers have been proposed [1]-[5], such as, representing them with real numbers [6]-[12]. The results of studies on ranking fuzzy numbers have been used in application areas such as decision making [13]-[22].

In order to rank fuzzy numbers, one fuzzy number needs to be evaluated and compared to the others. In this paper, the evaluation of fuzzy number is implemented by integral of all distances which are dot to lines, in which, lines are obtained by interval numbers of $\alpha$-cut set of the fuzzy number according to decomposition theorem. Based on the evaluation of fuzzy number, ranking fuzzy numbers could be finished. In order to illustrate the ranking method, numeric example is shown, and for the comparative study, our method is compared with four existing ranking methods. As an example of potential applications, the proposed method is applied to game with Fuzzy Profit.

\section{Interval measures and its ranking}

Generally, an interval number is represented as $A\left(a_{1}, a_{2}\right)=\left[a_{1}, a_{2}\right]$, where, $a_{1}, a_{2}$ respectively act as the interval number $A\left(a_{1}, a_{2}\right)$ and satisfy: $a_{1} \leq a_{2}$; especially, the interval number $A\left(a_{1}, a_{2}\right)$ is represented as the real number $a_{1}$ if and only if $a_{1}=a_{2}$. Let $F(R)=\left\{A\left(a_{1}, a_{2}\right) \mid a_{1} \leq\right.$ $\left.a_{2}, a_{1}, a_{2} \in R\right\}$ represents the interval numbers set. $\forall A\left(a_{1}, a_{2}\right), B\left(b_{1}, b_{2}\right) \in F(R)$,

$$
A\left(a_{1}, a_{2}\right)=B\left(b_{1}, b_{2}\right) \Longleftrightarrow a_{1}=b_{1}, a_{2}=b_{2} .
$$

Figure 1 describes the line segments between the two axis of the rectangular cartesian coordinates, $\left(a_{1}, 0\right)$ and $\left(0, a_{2}\right)$ are respectively used to denote the two ends of the line $l_{a_{1} a_{2}}$, and

$$
\begin{aligned}
l_{a_{1} a_{2}}=l_{b_{1} b_{2}} & \Longleftrightarrow\left(a_{1}, 0\right)=\left(b_{1}, 0\right),\left(0, a_{2}\right)=\left(0, b_{2}\right) \\
& \Longleftrightarrow a_{1}=b_{1}, a_{2}=b_{2} .
\end{aligned}
$$

For the line $l_{a_{1} a_{2}}$ and the interval number $A\left(a_{1}, a_{2}\right)$, the following correspondence relation holds.

Theorem 1 There exists a bijection between $L$ and $F(R)$. Where, $L=\left\{l_{a_{1} a_{2}} \mid l_{a_{1} a_{2}} \in \vec{L}, a_{1} \leq a_{2}\right\}$, and $\vec{L}$ denotes the set of segments between the two axis.

\section{Proof Let}

$$
\begin{array}{ll}
f: & L \longrightarrow F(R) \\
& l_{a_{1} a_{2}} \longrightarrow\left[a_{1}, a_{2}\right]=f\left(l_{a_{1} a_{2}}\right)
\end{array}
$$

1) $\forall l_{a_{1} a_{2}}, l_{a_{3} a_{4}}$, if $l_{a_{1} a_{2}}=l_{a_{3} a_{4}}$, then $a_{1}=a_{3}$, and $a_{2}=a_{4}$, therefore, $f\left(l_{a_{1} a_{2}}\right)=\left[a_{1}, a_{2}\right]=\left[a_{3}, a_{4}\right]=$ $f\left(l_{a_{3} a_{4}}\right)$, that is, $f$ is a mapping from $F(R)$ to $L ; 2) \forall l_{a_{1} a_{2}}, l_{b_{1} b_{2}} \in L$. If $l_{a_{1} a_{2}} \neq l_{b_{1} b_{2}}$, then $a_{1} \neq a_{2}$ or $a_{1} \neq a_{2}$, that is, $\left[a_{1}, a_{2}\right] \neq\left[b_{1}, b_{2}\right]$, so, 
$f$ is a single-valued mapping from $L$ to $F(R) ; 3$ ) $\forall\left[a_{1}, a_{2}\right] \in F(R), a_{1} \leq a_{2}$, then the segment identified by $\left(a_{1}, 0\right)$ and $\left(0, a_{2}\right)$ satisfies the condition of $L$, that is, $l_{a_{1} a_{2}} \in L, f$ is a surjective mapping from $L$ to $F(R)$. So $f$ is a bijection from $F(R)$ to $L$.

Definition 1 The measure of interval number $A\left(a_{1}, a_{2}\right)\left(=f\left(l_{a_{1} a_{2}}\right)\right)$ is denoted as follow:

$$
d_{O A}=d_{l_{a_{1} a_{2}}}=\frac{\sqrt{2}\left|a_{1} a_{2}\right|}{\sqrt{a_{1}^{2}+a_{2}^{2}}}
$$

Proposition 1 Let $A\left(a_{1}, a_{2}\right)$ be interval number and $a_{1} \neq 0, a_{2} \neq 0$, then:

1. if $a_{1}=a_{2}$ then $d_{O A}=a_{1}$;

2. when the left end point of the interval number $A\left(a_{1}, a_{2}\right)$ is fixed, $d_{O A}$ is a monotone increasing function about the right end point $a_{2}$, and $d_{O A} \rightarrow \sqrt{2}\left|a_{1}\right|\left(a_{2} \rightarrow+\infty\right)$;

3. when the right end point of the interval number $A\left(a_{1}, a_{2}\right)$ is fixed, $d_{O A}$ is a monotone increasing function about the left end point $a_{1}$, and $d_{O A} \rightarrow \sqrt{2}\left|a_{1}\right|\left(a_{1} \rightarrow-\infty\right) ;$

4. the measure of all the interval numbers cutting the circular which center lies at $O$ and radius is $r$ are equal, i.e., $d_{O A}=r$.

Proof These can easily be proved by (1).

Theorem 2 A relation $\sim$ on $L$ is defined as follow: $\forall l_{1}, l_{2} \in L$,

$$
l_{1} \sim l_{2} \Leftrightarrow d_{l_{1}}=d_{l_{2}},
$$

then $\sim$ is an equivalence relation on $L$.

Proof (1) $\forall l_{1} \in L$, due to $d_{l_{1}}=d_{l_{1}}$, then $l_{1} \sim l_{1}$;

(2) $\forall l_{1} \in L, \forall l_{2} \in L$, due to $d_{l_{1}}=d_{l_{2}}$, then $l_{1} \sim l_{2}$

(3) $\forall l_{1} \in L, \forall l_{2} \in L, \forall l_{3} \in L, l_{1} \sim l_{2}, l_{2} \sim l_{3} \Rightarrow$ $d_{l_{1}}=d_{l_{2}}, d_{l_{2}}=d_{l_{3}} \Rightarrow d_{l_{1}}=d_{l_{3}} \Rightarrow l_{1} \sim l_{3}$, so $\sim$ is an equivalence relation.

Based on the equivalence relation $\sim$, we can categorize $L$, that is, two lines is in the same equivalent class if their measures are equal. So $[r]$ can be used to represent the equivalence class of $L$, and

$$
L=\bigcup[r], r \in[0,+\infty),
$$

where, $\forall l_{a_{1} a_{2}} \in[r]$ means that the measure of the line is $R$. Based on the measure of the line, distance between two interval numbers can be defined formally as follows

$d_{A\left(a_{1}, a_{2}\right) B\left(a_{3}, a_{4}\right)}=d_{l_{1} l_{2}}=\left|d_{O A}-d_{O B}\right|=\left|d_{l_{1}}-d_{l_{2}}\right|$

For $d_{A\left(a_{1}, a_{2}\right) B\left(a_{3}, a_{4}\right)}$, the follows property holds
Proposition 2 let $A\left(a_{1}, a_{2}\right)$ and $B\left(b_{1}, b_{2}\right)$ are two interval numbers then $d_{A\left(a_{1}, a_{2}\right) B\left(a_{3}, a_{4}\right)}$ satisfies follows:

1. $d_{A\left(a_{1}, a_{2}\right) A\left(a_{1}, a_{2}\right)}=0$;

2. $d_{A\left(a_{1}, a_{2}\right) B\left(a_{3}, a_{4}\right)}=d_{B\left(a_{3}, a_{4}\right) A\left(a_{1}, a_{2}\right)}$;

3. $d_{A\left(a_{1}, a_{2}\right) C\left(a_{5}, a_{6}\right)} \leq d_{A\left(a_{1}, a_{2}\right) B\left(a_{3}, a_{4}\right)}+$ $d_{B\left(a_{3}, a_{4}\right) C\left(a_{5}, a_{6}\right)}$.

So it can be used to act as the Haudorff-distance from A to B. We can see from (1).

1. When two interval numbers $A\left(a_{1}, a_{2}\right)$, $B\left(b_{1}, b_{2}\right)$ degenerate into two real numbers, their distance $d_{A B}=\left|a_{1}-b_{1}\right|$;

2. The distance from the atom to interval $\left(a_{1},+\infty\right)$ is $\sqrt{2} a_{1}$;

3. The distance from the interval number $\left(a_{1},+\infty\right)$ to $\left(a_{2},+\infty\right)$ is $\sqrt{2}\left|a_{1}-a_{2}\right|$;

4. For two interval numbers $A\left(a_{1}, a_{2}\right), B\left(b_{1}, b_{2}\right)$, when $b_{1}>a_{2}$, then $d(O B)>d(O A)$.

The measure of interval number acts as a criterion about interval number comparison.

Definition 2 Let $A\left(a_{1}, a_{2}\right), B\left(b_{1}, b_{2}\right)$ are two interval numbers, $A\left(a_{1}, a_{2}\right) \leq B\left(b_{1}, b_{2}\right) \Leftrightarrow d(O A) \leq$ $d(O B)$.

Denotes $[r]=\left\{A\left(a_{1}, a_{2}\right) \in F(R) \mid d(O A)=r\right\}$.

Proposition 3 If $A\left(a_{1}, a_{2}\right) \in\left[r_{1}\right], B\left(b_{1}, b_{2}\right) \in\left[r_{2}\right]$ then $A\left(a_{1}, a_{2}\right) \leq B\left(b_{1}, b_{2}\right) \Leftrightarrow r_{1} \leq r_{2}$

Proposition 4 Let $A\left(a_{1}, a_{2}\right)$ and $B\left(b_{1}, b_{2}\right)$ be two close interval numbers, and $a_{1} \geq 0, b_{1} \geq 0$ :

1. if $a_{2} \leq b_{1}$ then $A\left(a_{1}, a_{2}\right) \leq B\left(b_{1}, b_{2}\right)$;

2. if $a_{1} \leq b_{1}, a_{2} \leq b_{2}$, then $A\left(a_{1}, a_{2}\right) \leq B\left(b_{1}, b_{2}\right)$.

Proof (1) Because $0 \leq a_{1} \leq a_{2}$, therefore $d(O A)=$ $\frac{\sqrt{2} a_{1} a_{2}}{\sqrt{a_{1}^{2}+a_{2}^{2}}} \leq \sqrt{2} a_{2}$. Because $0 \leq b_{1} \leq b_{2}$, therefore $d(O B)=\frac{\sqrt{2} b_{1} b_{2}}{\sqrt{b_{1}^{2}+b_{2}^{2}}} \geq \sqrt{2} b_{1}$, therefore if $a_{2} \leq b_{1}$ then $d(O A) \leq d(O B)$, that is $A\left(a_{1}, a_{2}\right) \leq B\left(b_{1}, b_{2}\right)$;

(2) Because $a_{1} \leq b_{1}, a_{2} \leq b_{2}$, therefore $\frac{1}{a_{1}^{2}}+$ $\frac{1}{a_{2}^{2}} \geq \frac{1}{b_{1}^{2}}+\frac{1}{b_{2}^{2}} \Rightarrow \frac{a_{1}^{2} a_{2}^{2}}{a_{1}^{2}+a_{2}^{2}} \leq \frac{b_{1}^{2} b_{2}^{2}}{b_{1}^{2}+b_{2}^{2}}$, so, $A\left(a_{1}, a_{2}\right) \leq$ $B\left(b_{1}, b_{2}\right)$.

\section{Ranking the fuzzy numbers}

Based on decomposition theorem of fuzzy set and central defuzzified method, the measure of interval number can be extended to become the measure of fuzzy number. Based on the measure of fuzzy number, ranking fuzzy numbers can be finished. This section mainly discusses ranking General Left Right Fuzzy Numbers (GLRFN). 
Definition 3 [12] Let $\widetilde{A}\left(a_{1}, a_{2}, a_{3}, a_{4}\right)$ be $a$ GLRFN. Its membership function is:

$$
\mu_{\widetilde{A}}(x)= \begin{cases}L(x), & \text { if } a_{1} \leq x<a_{2} \\ 1, & \text { if } a_{2} \leq x<a_{3} \\ R(x), & \text { if } a_{3} \leq x<a_{4} \\ 0, & \text { otherwise }\end{cases}
$$

Where, $L(x)$ is right-continuous strict monotonic increasing function and $0 \leq L(x) \leq 1 ; R(x)$ is left-continuous strict monotonic decreasing function, $0 \leq R(x) \leq 1, a_{1}<a_{2} \leq a_{3}<a_{4}$.

$\forall \alpha \in[0,1]$, the $\alpha$ - cuts interval of $u_{\widetilde{A}}(x)$ is:

$$
I_{\widetilde{A}}(\alpha)=\left[L^{-1}(\alpha), R^{-1}(\alpha)\right],
$$

especially, if $\alpha=1$, then:

$$
I_{\widetilde{A}}(1)=\left[L^{-1}(1), R^{-1}(1)\right]=\left[a_{2}, a_{3}\right] .
$$

The distance from $O$ to $I_{\widetilde{A}}(\alpha)$ is:

$$
d_{I_{\tilde{A}}}=\frac{\sqrt{2}\left|L^{-1}(\alpha) R^{-1}(\alpha)\right|}{\sqrt{L^{-1}(\alpha)^{2}+R^{-1}(\alpha)^{2}}} .
$$

Based on the fuzzy set theory(FST), we can obtain the measure of a GLRFN: $\widetilde{A}\left(a_{1}, a_{2}, a_{3}, a_{4}\right)$ as follows:

$$
\begin{aligned}
R_{\widetilde{A}} & =\frac{\int_{0}^{1} \alpha d_{I_{A}(\alpha)} d \alpha}{\int_{0}^{1} \alpha d \alpha} \\
& =\frac{\sqrt{2} \int_{0}^{1} \frac{\alpha\left|L^{-1}(\alpha) R^{-1}(\alpha)\right|}{\sqrt{L^{-1}(\alpha)^{2}+R^{-1}(\alpha)^{2}}} d \alpha}{\int_{0}^{1} \alpha d \alpha} .
\end{aligned}
$$

Based on (6), it can be gained: fuzzy number $\widetilde{A}\left(a_{1}, a_{2}, a_{3}, a_{4}\right)$ correspondence real number.

Especially, when $\widetilde{A}\left(a_{1}, a_{2}, a_{3}, a_{4}\right)$ is a trapezoidal fuzzy number, that is, $a_{1}<a_{2}<a_{3}<a_{4}$ and

$$
L(x)=\frac{x-a_{1}}{a_{2}-a_{1}}, \quad R(x)=\frac{a_{4}-x}{a_{4}-a_{3}},
$$

Based on (6) and (7), we can calculate $R_{\widetilde{A}}$ as follows:

$$
\begin{aligned}
R_{\widetilde{A}} & =\sqrt{2} \mid A\left(\frac{1}{3}\left[(1+2 B+D)^{\frac{3}{2}}-D^{\frac{3}{2}}\right]\right. \\
& +\left(2 E-2 D+5 B C-11 B^{2}\right) \\
& \cdot(\sqrt{1+2 B+D}-\sqrt{D}) \\
+ & (C-3 B) \sqrt{1+2 B+D} \\
+ & \frac{1}{2}\left(3 C D-5 B D-2 B E-5 B^{2} C+11 B^{3}\right) \\
& \cdot\left(\ln \frac{\sqrt{1+2 B+D}+1+B}{\sqrt{1+2 B+D}-1-B}-\ln \frac{\sqrt{D}+B}{\sqrt{D}-A}\right) \mid,
\end{aligned}
$$

where,

$$
\begin{aligned}
A & =\frac{\left(a_{2}-a_{1}\right)\left(a_{3}-a_{4}\right)}{\sqrt{\left(a_{2}-a_{1}\right)^{2}+\left(a_{3}-a_{4}\right)^{2}}}, \\
B & =\frac{a_{1}\left(a_{2}-a_{1}\right)-a_{4}\left(a_{3}-a_{4}\right)}{\left(a_{2}-a_{1}\right)^{2}+\left(a_{3}-a_{4}\right)^{2}}, \\
C & =\frac{a_{4}\left(a_{2}-a_{1}\right)+a_{1}\left(a_{3}-a_{4}\right)}{\left(a_{2}-a_{1}\right)\left(a_{3}-a_{4}\right)} \\
D & =\frac{a_{1}^{2}+a_{4}^{2}}{\left(a_{2}-a_{1}\right)^{2}+\left(a_{4}-a_{3}\right)^{2}}, \\
E & =\frac{a_{1} a_{4}}{\left(a_{2}-a_{1}\right)\left(a_{3}-a_{4}\right)}
\end{aligned}
$$

Based on (6), we can identify a mapping from the GLRFN $F(R)$ to $(0,+\infty)$ :

$$
\begin{gathered}
f: F(R) \rightarrow(0,+\infty), \\
\widetilde{A}\left(a_{1}, a_{2}, a_{3}, a_{4}\right) \rightarrow R_{\widetilde{A}} .
\end{gathered}
$$

Naturally, the ranking question can be convert into the comparison of the correspondence measure.

Definition 4 If $\widetilde{A}\left(a_{1}, a_{2}, a_{3}, a_{4}\right), \widetilde{B}\left(b_{1}, b_{2}, b_{3}, b_{4}\right) \in$ $F(R)$, let:

$$
\widetilde{A}\left(a_{1}, a_{2}, a_{3}, a_{4}\right) \leq \widetilde{B}\left(b_{1}, b_{2}, b_{3}, b_{4}\right) \Leftrightarrow R_{\widetilde{A}} \leq R_{\widetilde{B}} .
$$

\section{Numeric examples and com- parison with previous works}

In this subsection, in order to show how the proposed method works and how viewpoints affect the ranking result, two examples are presented according to [1].

Example 1 In Fig 1, (a), (b), (c), (d) and (e) respectively describe five kinds of fuzzy numbers.

The three fuzzy numbers in (a) are respectively $\widetilde{A_{1}}(0.4,0.5,1), \widetilde{A_{2}}(0.4,0.7,1), \widetilde{A_{3}}(0.4,0.9,1)$, then we have: $R_{\widetilde{A_{1}}}=3.17, R_{\widetilde{A_{2}}}=3.59, R_{\widetilde{A_{3}}}=3.48$, hence $\widetilde{A_{1}}<\widetilde{A_{3}}<\widetilde{A_{2}}$.

The two fuzzy numbers in (b) are respectively $\widetilde{A_{1}}(0.2,0.5,0.8), \widetilde{A_{2}}(0.4,0.5,0.6)$, then we have: $R_{\widetilde{A_{1}}}=3.61, R_{\widetilde{A_{2}}}=3.14$, hence $\widetilde{A_{2}}<\widetilde{A_{1}}$.

The three fuzzy numbers in (c) are respectively $\quad \widetilde{A_{1}}(0.5,0.7,0.9), \quad \widetilde{A_{2}}(0.3,0.7,0.9)$, $\widetilde{A_{3}}(0.3,0.4,0.7,0.9)$, then we have: $R_{\widetilde{A_{1}}}=3.51$, $R_{\widetilde{A_{2}}}=3.89, R_{\widetilde{A_{3}}}=3.67$, hence, $\widetilde{A_{1}}<\widetilde{A_{3}}<\widetilde{A_{2}}$.

The three fuzzy numbers in (d) are respectively $\widetilde{A_{1}}(0.3,0.5,0.8,0.9), \quad \widetilde{A_{2}}(0.3,0.5,0.9)$, $\widetilde{A_{3}}(0.3,0.5,0.7)$, then we have: $R_{\widetilde{A_{1}}}=3.03$, $R_{\widetilde{A_{2}}}=2.74, R_{\widetilde{A_{3}}}=3.06$, hence, $\widetilde{A_{2}}<\widetilde{A_{1}}<\widetilde{A_{3}}$. 


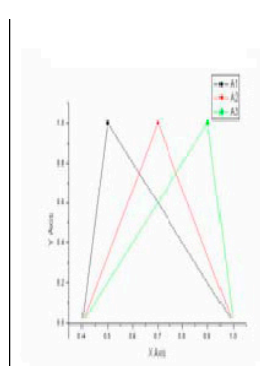

(a)

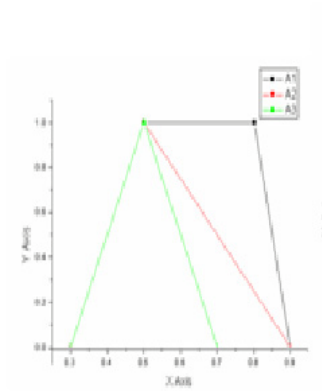

(d)

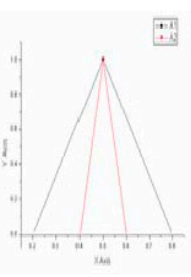

(b)

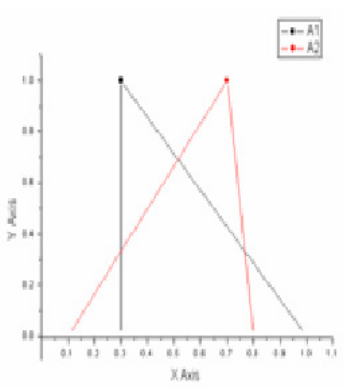

(e)
Fig. 1: Figures about fuzzy numbers

The two fuzzy numbers in (e) are respectively $\widetilde{A_{1}}(0.3,0.3,1), \widetilde{A_{2}}(0.1,0.7,0.8)$, then we have: $R_{\widetilde{A_{1}}}=3.86, R_{\widetilde{A_{2}}}=4.27$, hence, $\widetilde{A_{2}}<\widetilde{A_{1}}$.

Table 4 is the result of comparison with previous works, in which, the methods refer to [15], [16], [17], [4], [19], [12], [18], [10] and [6], respectively, the content of Table 4 partly comes from [6].

In the following, we will apply the proposed ranking method in the realistic problem - the Game with Crisp Profit. To describe the decision-making problem, we will present an example with crisp profit and loss. The description of the problem is as follows: there are two players-A and B. Player A can choose one action among $a_{1}, a_{2}, a_{3}$ and player $\mathrm{B}$ among $b_{1}, b_{2}, b_{3}$. When the game starts, player $\mathrm{A}$ chooses first, and then B chooses. Each player's selection. A's profit and loss are the opposite of B's. Table I shows A's profit or loss.Thus, for example, if $\mathrm{A}$ chooses action $a_{1}$ and $\mathrm{B}$ chooses $b_{1}$, then $\mathrm{A}$ will get a profit of 5 and $\mathrm{B}$ a profit of -5 , i.e., a loss of 5 . By this rule, $\mathrm{A}$ and $\mathrm{B}$ try to maximize their own profits.then which action should A choose to maximize his profit? Let us analyze A's profit in the case where $\mathrm{A}$ chooses $a_{1}$. Which action will $\mathrm{B}$ choose in this case? If $\mathrm{B}$ chooses $b_{1}$, $\mathrm{B}$ will get
Table 1: Player A's Fuzzy Profit and loss

\begin{tabular}{|c||c|c|c|}
\hline & $b_{1}$ & $b_{2}$ & $b_{3}$ \\
\hline \hline$a_{1}$ & 5 & -5 & -4 \\
\hline$a_{2}$ & -4 & 7 & 0 \\
\hline$a_{3}$ & -4 & 7 & 0 \\
\hline
\end{tabular}

Table 2: Player A's Fuzzy Profit and loss

\begin{tabular}{|l||c|c|c|}
\hline & $b_{1}$ & $b_{2}$ & $b_{3}$ \\
\hline \hline$a_{1}$ & $F_{11}$ & $F_{12}$ & $F_{13}$ \\
\hline$a_{2}$ & $F_{21}$ & $F_{22}$ & $F_{23}$ \\
\hline$a_{3}$ & $F_{31}$ & $F_{32}$ & $F_{33}$ \\
\hline
\end{tabular}

-5 (because in this case A will get 5 ); if B chooses $b_{2}$, the profit is 5 ; and if $b_{3}$ then the profit is 4 . Alternative $b_{2}$ will give $\mathrm{B}$ the maximum profit. Thus, $\mathrm{B}$ will choose $b_{2}$, and $\mathrm{A}$ will lose 5 . In a similar manner, if $\mathrm{A}$ chooses $a_{2}$, then $\mathrm{B}$ chooses $b_{1}$. As the result of this selection, $\mathrm{A}$ gets -4 . If A chooses $a_{3}, \mathrm{~B}$ will choose $b_{3}$ and $\mathrm{A}$ will get -8 . Thus, considering these possibilities, in order to maximize A's profit, A should choose $a_{2}$, which will give $\mathrm{A}$ at least a guaranteed profit of -4 . B. Game with Fuzzy profit.

In cases where profit and loss are described with crisp numbers, A's choice can be simply determined. However, if profit and loss are fuzzily described, the optimal selection is not easy to determine. As mentioned in Section I, it is difficult to determine whether a fuzzy number is larger or smaller than another and the evaluations of fuzzy numbers may easily be affected by evaluation viewpoints which may differ from person to person. Thus, it is difficult to predict which selection a player will make. For example, what is the largest of the two fuzzy numbers in Fig.4? Which does an optimistic or a pessimistic player choose? In Fig.4, fuzzy number has a large width, so it includes large as well as small numbers. But, in the case of $F_{1}$, the width is small and includes only medium-sized numbers.

Thus, if a player is optimistic, then that player will choose $F_{1}$ because it has the values larger than $F_{2}$. On the other hand, if a player is pessimistic, that player will choose $F_{2}$ because $F_{1}$ has the values

Table 3: Player A's Fuzzy Profit and loss

\begin{tabular}{|l||c|c|c|}
\hline & $b_{1}$ & $b_{2}$ & $b_{3}$ \\
\hline \hline$a_{1}$ & 3.17 & 3.59 & 3.48 \\
\hline$a_{2}$ & 3.51 & 3.89 & 3.67 \\
\hline$a_{3}$ & 3.03 & 2.74 & 3.06 \\
\hline
\end{tabular}


Table 4: Comparison with previous works

\begin{tabular}{|c|c|c|c|c|c|c|c|c|c|c|c|c|c|}
\hline Methods & $A_{1}$ & $A_{2}$ & $A_{3}$ & $A_{1}$ & $A_{2}$ & $A_{1}$ & $A_{2}$ & $A_{3}$ & $A_{1}$ & $A_{2}$ & $A_{3}$ & $A_{1}$ & $A_{2}$ \\
\hline Yager $F_{1}$ & 0.76 & 0.7 & 0.63 & 0.5 & 0.5 & 0.7 & 0.63 & 0.57 & 0.62 & 0.56 & 0.5 & 0.61 & 0.53 \\
\hline$F_{2}$ & 0.9 & 0.76 & 0.66 & 0.61 & 0.54 & 0.75 & 0.75 & 0.75 & 0.81 & 0.64 & 0.58 & 0.66 & 0.69 \\
\hline$F_{3}$ & 0.8 & 0.7 & 0.6 & 0.6 & 0.5 & 0.7 & 0.65 & 0.57 & 0.62 & 0.54 & 0.5 & 0.58 & 0.56 \\
\hline Bass & 1 & 0.74 & 0.6 & 1 & 1 & 1 & 1 & 1 & 1 & 1 & 1 & 0.84 & 1 \\
\hline Baldwin 1 & 0.42 & 0.33 & 0.3 & 0.27 & 0.27 & 0.37 & 0.27 & 0.27 & 0.45 & 0.37 & 0.27 & 0.42 & 0.33 \\
\hline and $g$ & 0.55 & 0.4 & 0.34 & 0.3 & 0.24 & 0.42 & 0.35 & 0.35 & 0.53 & 0.4 & 0.28 & 0.44 & 0.37 \\
\hline Guild $\mathrm{r}$ : a & 0.28 & 0.23 & 0.22 & 0.2 & 0.23 & 0.27 & 0.19 & 0.19 & 0.31 & 0.28 & 0.21 & 0.34 & 0.24 \\
\hline Kerre & 1 & 0.86 & 0.76 & 0.91 & 0.91 & 1 & 0.91 & 0.75 & 1 & 0.85 & 0.75 & 0.96 & 0.89 \\
\hline Jain $\mathrm{k}=1$ & 0.9 & 0.76 & 0.66 & 0.73 & 0.67 & 0.82 & 0.82 & 0.82 & 0.9 & 0.69 & 0.64 & 0.66 & 0.69 \\
\hline $\mathrm{k}=2$ & 0.84 & 0.65 & 0.54 & 0.6 & 0.48 & 0.71 & 0.71 & 0.71 & 0.82 & 0.56 & 0.45 & 0.53 & 0.51 \\
\hline $\mathrm{k}=0.5$ & 0.95 & 0.86 & 0.78 & 0.83 & 0.8 & 0.89 & 0.89 & 0.89 & 0.94 & 0.8 & 0.77 & 0.78 & 0.81 \\
\hline Dubois PD & 1 & 0.74 & 0.6 & 1 & 1 & 1 & 1 & 1 & 1 & 1 & 1 & 0.84 & 1 \\
\hline and PSD & 0.74 & 0.23 & 0.16 & 0.73 & 0.24 & 0.5 & 0.5 & 0.5 & 0.8 & 0.2 & 0 & 0.54 & 0.46 \\
\hline Prade ND & 0.63 & 0.38 & 0.18 & 0.27 & 0.76 & 0.67 & 0.35 & 0 & 0.5 & 0.5 & 0.5 & 0.54 & 0.46 \\
\hline NSD & 0.26 & 0 & 0 & 0 & 0 & 0 & 0 & 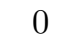 & 0 & 0 & 0 & 0 & 0.16 \\
\hline Lee U.m & 0.76 & 0.7 & 0.63 & 0.5 & 0.5 & 0.7 & 0.63 & 0.57 & 0.62 & 0.56 & 0.5 & 0.61 & 0.53 \\
\hline and U.G & - & - & - & 0.12 & 0.04 & - & - & - & - & - & - & - & - \\
\hline Li P.m & 0.8 & 0.7 & 0.6 & 0.5 & 0.5 & 0.7 & 0.65 & 0.58 & 0.63 & 0.55 & 0.5 & 0.53 & 0.58 \\
\hline P.G & - & - & - & 0.09 & 0.03 & - & - & - & - & - & - & - & - \\
\hline Fortemps $F_{0}$ & 0.8 & 0.7 & 0.6 & 0.5 & 0.5 & 0.7 & 0.65 & 0.58 & 0.63 & 0.55 & 0.5 & 0.49 & 0.61 \\
\hline $\operatorname{Tran} D_{\max }^{x}$ & 0.19 & 0.31 & 0.44 & 0.51 & 0.5 & 0.3 & 0.34 & 0.46 & 0.4 & 0.47 & 0.5 & 0.57 & 0.36 \\
\hline$D_{\min }^{x}$ & 0.84 & 0.7 & 0.57 & 0.51 & 0.5 & 0.7 & 0.67 & 0.59 & 0.65 & 0.54 & 0.5 & 0.45 & 0.67 \\
\hline$D_{\max }^{1}$ & 0.23 & 0.32 & 0.42 & 0.51 & 0.5 & 0.31 & 0.37 & 0.45 & 0.4 & 0.46 & 0.5 & 0.53 & 0.42 \\
\hline$D_{\min }^{1}$ & 0.81 & 0.71 & 0.61 & 0.51 & 0.5 & 0.7 & 0.66 & 0.59 & 0.64 & 0.56 & 0.5 & 0.51 & 0.63 \\
\hline Our Method & 3.17 & 3.59 & 3.48 & 3.61 & 3.14 & 3.51 & 3.88 & 3.66 & 3.02 & 2.73 & 3.06 & 3.85 & 4.26 \\
\hline
\end{tabular}

smaller than $F_{2}$. Thus, a decision-making method which considers these factors is needed. In this section, in order to deal with these problems, a decision-making using our new ranking method is proposed. A decision-making method with fuzzy profit and loss is presented. It is assumed that A's fuzzy numbers are depended the Fig(a), (c) and (d) in above example an A's profit and loss are showed in Table 3. It is assumed that A's fuzzy numbers are depended the Figure(a), (c) and (d) in above example an A's profit and loss are showed in Table 3. In this case, which should A choose to maximize the profit? According to above discussion, it is easily obtained that the answer is that A should choose $a_{3}$ and B should choose $b_{2} \mathrm{~F}(32)=2.74$.

\section{Conclusion}

In this paper, a new method for ranking fuzzy numbers, which is based on the distance of dot to lines, is proposed. Based on decomposition theorem of fuzzy set, integral of all distances is used as evaluation of fuzzy number. Comparing our method with previous works about ranking fuzzy number are done in this paper.

\section{Acknowledgement}

This work is supported by the Excellent Young Foundation of Sichuan Province (Grant No.06ZQ026-037), the National Natural Science Foundation of China (60474022), the Special Research Funding to Doctoral Subject of Higher Education Institutes in China (Grant No.20060613007) and a Project Supported by Scientific Research Fund of SiChuan Provincial Education Department(Grant No.2005A121, 2006A084).

\section{References}

[1] G. Bortolan, R. Degani, A review of some method for ranking fuzzy sets. Fuzzy Sets and Systems, 15:1-19, 1985.

[2] M. G. Iskander, Comparison of fuzzy numbers using possibility programming: comments and new concepts. Computers and Mathematics with Applications, 43:833-840, 2002. 
[3] Y. X. Yuan, Criteria for evaluating fuzzy ranking method. Fuzzy Sets and Systems, 44:139157, 1991.

[4] X. Wang, E. E. Kerre, Reasonable properties for the ordering of fuzzy quantities(I). Fuzzy Sets and Systems, 118:375-385, 2001.

[5] X. Wang, E. E. Kerre. Reasonable properties for the ordering of fuzzy quantities(II). Fuzzy Sets and Systems, 118:387-405, 2001.

[6] L. Tran, L. Ducksteinb, Comparison of fuzzy numbers using a fuzzy distance measure. Fuzzy Sets and Systems, 130:331-341, 2002.

[7] W. Voxman, Some remarks on distance between fuzzy numbers. Fuzzy Sets and Systems, 100:353-365, 1998.

[8] C. H. Cheng, A new approach for ranking fuzzy numbers by distance method. Fuzzy Sets Syst, 95:307-317, 1998.

[9] T. S. Liou, M. J. Wang, Ranking fuzzy numbers with integral value. Fuzzy Sets Syst, 50:247-255, 1992.

[10] P. Fortemps, M. Roubens, Ranking and defuzzification methods based on area compensation. Fuzzy Sets Syst, 82:319-330, 1996.

[11] K. P. Yoon, A probabilistic approach to rank complex fuzzy numbers. Fuzzy Sets Syst, 80:167-176, 1996.

[12] D. Dubois, H. Prade, Ranking of fuzzy numbers in the setting of possibility theory. Inform. Sci., 30:183-224, 1983.

[13] K. Sengupta, Fuzzy preference and Orlovsky choice procedure. Fuzzy Sets Syst, 93:231-234, 1998.

[14] J. H. Lee and H. Lee-Kwang, Distributed and cooperative fuzzy controller for traffic intersection group. IEEE Trans. Syst., Man, Cybern., 29(C):263-271, 1999.

[15] R. R. Yager,A procedure for ordering fuzzy subsets of the unit interval. Information science ,24:143-161, 1981.

[16] S. M. Bass, H. Kwakernaak, Rating and ranking of multiple-aspect alternatives using fuzzy sets. Automatics, 13:47-58, 1977.

[17] J. F. Baldwin, N. C. F. Guild, Comparison of fuzzy sets on the same decision space. Fuzzy Sets and Systems, 2: 213-233, 1979.

[18] R. J. Li, E. S. Lee, Ranking fuzzy numbersAcomparison,Proc NAFIPS Workshop, West Lafa yet. Fuzzy Sets and Systems, 143:168-204, 1987.

[19] R. Jain, Decision-making in the presence of fuzzy variables.IEEE Trans.systems.Man Cybernet.,6:689-703, 1976.

[20] H. Lee-Kwang, Jee-Hyong Lee, A Method for
Ranking Fuzzy Numbers and Its Application to Decision-Making. IEEE Transactions On Fuzzy Systems ,7(6):677-685, 1999.

[21] P. Zheng, Y. L. Zhong, A New Aggregation operator of Languistic Information And Its Properties. IEEE International Conference on Granular Computing, pp: 486-489, 2006.

[22] Z. Xu, Induced uncertain linguistic OWA operators applied to group decision making. Information Fusion, 7:231-238, 2006. 